\title{
CP AND B PHYSICS: PROGRESS AND PROSPECTS
}

\author{
J. D. Bjorken \\ Stanford Linear Accelerator Center \\ Stanford University, Stanford, California 94309
}

\begin{abstract}
This summary of the 2nd International Conference on $B$ Physics and CP Violation (Honolulu, 24-27 March, 1997) contains, in addition to what is implied in the title, some extended remarks on the limitations of theory as well as speculations regarding nonperturbative enhancement of decay modes of the class $b \rightarrow s+$ charmless hadrons.
\end{abstract}

\section{Generalities}

It is a privilege for me, but something of a disadvantage otherwise, that I give this summary. I have not been active in $B$-physics for some time. The last time I did review the subject was in the mid 1980s 1 . So, as an outsider, I will neither try to provide a compendium of the many interesting contributions, nor a comprehensive summary of the field. There will be few if any detailed quotations of results or exhibits of plots from the contributors to the meeting, and this overview will most likely be rather unbalanced.

On the other hand, there are some marginal advantages to being an outsider. I perhaps can feel the changes which have been occurring within the field in the last decade more easily than those of you who are embedded within it full-time. And unlike some of our subfields which feel all too static, there really is a dynamic change in the $\mathrm{CP} / b$-quark business. The primary driving force in physics is the technological progress, next the experiments which benefit from it, and finally the theory. In all three areas there have been most impressive advances. At DESY with DORIS/ARGUS, but especially at Cornell with CESR/ CLEO, we have seen the storage ring performance steadily improve and the detector capability enhanced. CDF, SLD, and the LEP experiments have discovered $B$-physics and have done much more with it than at least I anticipated would be done. The asymmetric electron-positron collider idea emerged and has gone from the $R \& D$ phase to the construction phase; it of course provides great promise for CP-violation-level $B$-physics. The new round of kaon-decay experiments are extraordinary in the statistical power and level of precision being attained. Charm physics at Fermilab and at CERN in fixed-target mode has been remarkably productive not only in the data acquired but also in the technological advances which those experiments pioneered. The technological changes most important are the microvertex detectors and the high rate, large bandwidth data acquisition systems. And the beautiful photon detection ca- 
pability at CESR via CsI (and at L3 with BGO) has made a huge difference. And at long last the ring-imaging Cerenkov technique seems to have matured.

At present there is a very big data base of high quality. The CKM parameters are already measured quite well, although we all hunger for still more accuracy. And on the theoretical side, there has emerged what I used to call Wisgur, in honor (???) of the originators Wise and Isgure, and which is now called Heavy Quark Effective Theory (HQET). This development transcended the model-building approach which preceded it, and provides a solid foundation for analysis of semileptonic decays and is an important tool for a variety of other problems.

And the community has grown into a very large one, with very high professional standards. I hope that what I perceive is not just the progress in producing transparencies, so impressive that it becomes impossible to doubt the truth of what is displayed upon them.

Ten years ago the big question was whether $\mathrm{CP}$ violation in the $B$ system could ever be seen 3 . Now the question has simply become who will see it first. The race is on, and my (conservative) guess of completion time is no later than 2001 or so. Coming in first is rightly important to the participants in the race. But it has to be emphasized that the end of the race is just the beginning of the field - the first measurements must be supplemented by many others to get the big picture of what any one measurement really means. So losers really won't lose. Everyone should win, because the variety of CP violating phenomena which becomes accessible at a level of difficulty not far beyond the discovery mode is very large. There is plenty of room for everybody.

Nowadays, the issues in the $B$ business seem to have become sharply defined. One sees in the projections of new initiatives quite precise specifications of what must be accomplished, e.g. the precision to which $\sin 2 \beta$ or $x_{s}$ will be measured, etc. 1 It looks almost like engineering. Is it really true that the future of $B$-physics will be another round of LEP I Yellow-book physics, with many fine results of exquisite precision, but constrained within standard-model expectations? Of course nobody knows. This could happen, and it often seems that this is the standard presumption. On the other hand there might be quite strong shocks to the standard picture. In particular, one might ask

1. Is the unitarity triangle not?

or

2. Is the unitarity triangle not enough?

or, on the other hand,

3. Is the unitarity triangle right? 
Now for the explanations of what is meant, in turn:

- Maybe the unitarity triangle is not an interesting one, i.e. it is degenerate. Perhaps it will be discovered that there exists an unexpected discrete symmetry, like the occasional discrete symmetries entertained for reasons of expediency by SUSY model-builders and others, but this one immediately accessible. This one could be called electroweak $T$ conservation, i.e. there does exist a basis where all CKM parameters are real. This is what is implied by the superweak theory and also by the aspon model, presented here by faul Frampton 5 . The data as presented at this meeting by Roger Forty 6 is only marginally consistent with this hypothesis, but it was emphasized by many that the systematic errors are dominated by theoretical uncertainty and cannot be treated reliably by standard statistical methods. I agree with this conventional wisdom. And since the main culprit in error production is $V_{t d}$, its measurement by $B_{d}$ and $B_{s}$ mixing (or $K \rightarrow \pi \nu \bar{\nu}$ ) seems to be the best way of improving the situation. But imagine the impact of the electroweak $T$-conserving scenario: the kaon experiments see no evidence for nonvanishing $\epsilon^{\prime}$, and everyone measures everything at the $B$-factories, with no trace of $\mathrm{CP}$ violation. Fantastically exciting!! It's the arrival of new, unexpected physics, not with a bang but with the whimpers of a thousand experimentalists.

- Maybe the CKM parametrization of CP violating effects is not enough, and that in addition to the standard sources of $\mathrm{CP}$ violation there are extra contributions. This could lead to inconsistent "determinations" of the angles $\alpha, \beta$, and $\gamma$. It could also lead to CP violation occurring where it is not expected to occur, such as in $\psi \eta, \psi \phi, b \rightarrow s \gamma$, etc. One moral to the story is to look everywhere for $\mathrm{CP}$ violation, even in places shunned by respectable theorists. Another moral is that it is important to measure the standard CKM parameters as redundantly as possible. Lincoln Wolfenstein seemed in his talk $\mathrm{U}$ to be somewhat distressed by the difficulties in sorting things out in this scenario. I can only wish that this were our problem.

- Maybe there is a right angle in the unitarity triangle, in particular maybe $\gamma=90^{\circ}$. This is not in the same class of dramatic surprises as the previous two categories, but nevertheless an observed regularity of shape of the unitarity triangle might send a rather strong message. There is a small, elite right-angle club, consisting to the best of my knowledge of Berthold Stech and myself. Harald Fritsch qualifies as a corresponding member (email only), having also advocated a right angle 8 , but the wrong one $(\alpha)$. 
The idea is Stech's 9 and is based on the hypothesis that, in a basis where the up-quark mass matrix is diagonal, the down quark mass matrix has real diagonal elements and small imaginary-antisymmetric off-diagonal elements 10 . The smallness requirement is that second-order perturbation theory suffices in the calculation of CKM parameters. So it was a delight to witness the exhibit of the unitarity triangle by Forty, so consistent with Stech's hypothesis, even though the consistency is without much meaning (remember item 1 above!).

\section{Trouble List}

To me, it is more important to pay attention to the difficulties in the subject, rather than the successes - even in a summary like this. It is in thinking about the difficulties more than in celebrating the progress that advances are made. In this meeting, a variety of problems were mentioned. None are anywhere near a crisis stage, but many are worth some serious attention. What follows is, first, a trouble list that I have put together, but only from my limited exposure to the subject at this meeting, with very brief descriptive remarks and references to the source material. This will be followed by some elaborations on the first five items on the list:

- Limitations of the theory:

Already in the discussion of the unitarity triangle above 6 , we have encountered the regrettable fact that theoretical, not experimental errors dominate. Lattice QCD provides valuable assistance on some problems, but its range of applicability js rather limited. And while HQET provides an excellent framework 11 for some of the theoretical questions, this is not true in general. Furthermore, the applicability of perturbative, short-distance, weak-coupling QCD is in my mind at best marginally defensible. More will be said about this later.

- Smallness of the semileptonic branching ratio and charm-counting:

The value of the semileptonic branching ratio has for a long time been a bit small for theory, suggesting an enhancement of some of the nonleptonic modes 21.13. The most attractive hypothesis is that it is the $c \bar{c} s$ class of final states that are enhanced, due to the small $Q$-value-and the possibility of resonances, etc. as the enhancement mechanism 12. This would imply a larger-than-expected number of charm hadrops produced per decay. But not enough is seen to satisfy the proponents 4 . 
- Magnitude of the $b \rightarrow s+$ charmless channels:

The charm-fraction problem has increased the focus on this class of channels, which are "gluonic penguin" and/or $c \bar{c}$ final-state annihilation channels. Standard PQCD estimates 15 put this branching ratio in the $1-2 \%$ range. For reasons of new-physics, Kagan would like an order of magnitude larger 13. And for me (as well as others 12 ) it is an interesting question whether nonperturbative QCDeffects could yield a larger value. As emphasized by Kagan and Dunietz 12.13 , measurement of the yield of energetic direct kaons (those not from charm decays) is a good way to get at this issue experimentally.

- Why so many $\eta^{\prime}$ 's in $B$ decays?

CLEO has presented 16 a variety of decay modes featuring $\eta^{\prime}$ 's. The branching ratio into $\eta^{\prime} K$ is about $7.5 \times 10^{-5}$, four times larger than the estimate of BSW from theory 17 . The inclusive yield into $K \eta^{\prime}$ and any number of additional pions, with the requirement that the $\eta^{\prime}$ lab momentum exceed $2 \mathrm{GeV}$, is an order of magnitude larger. What is the message, if any, from these new measurements? 18

- Why is the $\Lambda_{b}$ lifetime so short?

The $\Lambda_{b}$ lifetime appears to be only about $80 \%$ of the $B$-meson lifetimes 19. Short-distance, PQCD-based theory, according to Neubert 11 , will have a very hard time getting anything less than $90 \%$ for the ratio. At present, the measurements of semileptonic branching ratio of the $\Lambda_{b}$ give a lower number than for the mesons. The analysise is difficult, but does suggest that the increase in $\Lambda_{b}$ width originates in the nonleptonic-decay sector. The experimental numbers are still semi-soft, but hard enough to create some concern.

- Why do the parameters $a_{1}$ and $a_{2}$, which describe the relative importance of the so-called "color-allowed" and "color-suppressed" nonleptonic-decay four-fermion interaction terms, change their relative sign in ooing from charm decays (opposite sign) to bottom decays (same sign)? 21

Berthold Stech 22 expressed puzzlement on this problem; this again may be a signal that thinking that extends beyond the perturbative-QCD limit is required.

- Why is the (now well-observed) 23 width of $B$ into $K \pi$ larger than that into $\pi \pi$ ? 
The former contribution is in the penguin-induced category, while the latter can be estimated via factorization and knowledge of charmless semileptonic decays into $\pi \ell \nu$.

- Why is the hyperfine mass-splitting between $\Sigma_{b}^{*}$ and $\Sigma_{b}$ so large?

Heavy quark effective theory predicts 24 this splitting with confidence in terms of the corresponding hyperfine interval in the charm system. The central value emergent from the data25 is more than a factor of two too large. However, it is still possible to blame the data, not theory.

- What is the phenomenology of the nonresonant semileptonic decays of $B$ into $\left(D, D^{*}\right) \pi \ell \nu$ ?

The bookkeeping on the semileptonic $B$ decays now is good enough to determine that after $D, D^{*}$, and $D^{* *}$ final states are accounted for, there is still a coun fegf percent of branching ratio into the aforementioned channels 26.27.28.29.121. There is at present not good theoretical control on what the physics underlying those decay modes represents. Since these semileptonic decays are such a fundamental HQET playground, this represents a rather important challenge.

- What is one to do about "penguin pollution"?

There was quite a lot of discussion 30 of the problems created by interference of various $\mathrm{CP}$-violating penguin contributions with the more calculable ones, introducing difficult-to-anticipate strong-interaction phases into the analysis, and beclouding the extraction of accurate values of the CKM phases. This question to me is easy to answer. First, we should be so fortunate to have that problem. Experimentalists, please go out and observe these dirty $\mathrm{CP}$ violating processes, without inhibition!! (I hope and trust that this is totally unnecessary rhetoric.) Then let the further developments be data-driven. I fully expect that in many such cases the effects of the pollutants can and will be controlled by ideas on and measurements of related phenomena which are hard to anticipate in advance. There is nothing like some data to clear the mind.

\section{A few comments on the limitations of the theory:}

There were presented during the meeting a large number of theoretical contributions based on the techniques of short-distance, perturbative QCD. These go under the names operator-product expansion, PQCD, parton-hadron duality, etc. At the most basic level, they reflect the choice to describe the phenomenology at the parton level of quarks and gluons. 
It is not self-evident that this is appropriate. There are certainly successful applications of short-distance methods at energy scales less than $5 \mathrm{GeV}$. The total widths of charmonia come to mind, as well as the elegant analysis of tau decays to extract a value of the strong coupling constant. Also the whole subject of QCD sum rules resides in this energy range, although this technique involves art as well as science. However, success is far from guaranteed. During the meeting some of the theoretical discussion seemed to imply that one would be finding parton jets in the final states of $B$ decays. This will not happen. Electron-positron annihilation at $5 \mathrm{GeV}$ no doubt is initiated by a quark antiquark pair, but their hadronization leaves little trace of jettiness. True, the discovery experiment of "jettiness" did occur 31 at just this cms energy. But the evidence for jets was confined to the properties of the leading particle and was subtle at best. To good approximation at this energy the final state particle distribution is just phase space. For nonleptonic $B$ decays, originated by three, not two, partons, the situation has to be even more like phase space.

Then there is the question of "parton-hadron duality", the idea that on average the hadron-level behavior follows the parton-level behavior. An important application of this concept is to the important problem of decay widths. Again we can compare with the situation in electron-positron annihilation. If one compares the estimate of hadronic vacuum polarization, say, for spacelike virtual photons, to the perturbative estimate from quark loops and their radiative corrections, one gets quite good agreement. If one looks at the decay widths of the timelike photons, expressed in the famous parameter $R$, one must be more careful. In the entire range from zero energy up to $5.2 \mathrm{GeV}$, the estimate of $R$ from local parton-hadron duality is only reasonably reliable in the interval from 1.7 to $3.0 \mathrm{GeV}$ and from 4.6 to $5.2 \mathrm{GeV}$. Elsewhere there are resonances which locally distort the expected behavior by a factor of order unity (per relevant quark flavor). In the timelike region, one must average over an energy interval 32 to retrieve the short-distance results; this is just the uncertainty principle at work. In particular the energy interval should be large in comparison to the level spacing, which typically is of order a few hundred $\mathrm{MeV}$.

In semileptonic $B$ decays there is precious little phase space available, and three discrete levels dominate the final state. So there is a question of why HQET should work at all. The reason is that the best applications are independent of the assumption of parton-hadron duality, and that the physics is very simple. While one might expect corrections of the form

$$
\sim\left(\frac{\Lambda_{\mathrm{QCD}}}{m_{b}-m_{c}}\right)^{n}
$$


these do not appear 11, presumably because the underlying physics is so simple.

But in the nonleptonic decays, even for the $c \bar{u} d$ sector, the physics is less simple. Superposed (in space-time) upon the physics of the gentle, simple $b \rightarrow c$ semileptonic transition, is the physics of the hadronization of an outgoing $\bar{u}-d$ pair. If the pair go out in the same direction, they act as a small color dipole and do not create much disturbance 33. (This is now checked rather well experimentally 21.) If they go out in opposite directions, the more common case, then the color flux-tube that forms between them sits on top of the $b / c$ system plus light spectator-quark, and one cannot expect simplicity. So the hypothesis of "factorization", which essentially implies that these two subsystems do not talk to each other, becomes suspect. But even assuming factorization, the $\bar{u} d$ pair would have to have a mass large compared with 1.6 $\mathrm{GeV}$ before the local parton-hadron duality assumption becomes reliable, if one goes by the indications from electron-positron annihilation data.

So the bottom line is that even for estimating total nonleptonic widths, HQET may become HQIT, heavy-quark ineffective theory, and that one may have to take recourse to more explicit models. Many approaches deserve to be tried, and skepticism about any and all of them must be kept in the forefront.

There may be a larger role in B-decay phenomenology for LQET, light quark effective theory, than is in place at present. What is meant here is the long-distance limit of QCD expressed by the effective chiral action, based on constityent-quark and pion degrees of freedom (plus eventually a little bit of gluon 34 ). This is the formalism used for (but not limited to) chiral perturbation theory. A great deal of theoretical progress in this area has been made, both at a fundamental level and in its applications. There is a school of thomght that the LQET effective action, as catalogued by Gasser and Leutwyler 35, is already, if not actually derived, well-estimated from the first-principles QCD Lagrangian by integrating out the gluonic degrees of freedom, which at intermediate distances are assumed to be dominated by instanton contribution 36 . In particular, chiral symmetry breaking via this mechanism is claimed to occur 37 and be well understood, and many long-distance properties of hadrons well-estimated.

\section{A few comments on the experimental trouble list:}

As catalogued above in items 2 to 5 , there is a collection of bookkeeping issues which would be eased if the channels $b \rightarrow s+$ charmless hadrons were to be "surprisingly large", e.g. with branching ratio $5-15 \%$ instead of $1-2 \%$. Here, I address only the question of whether there can be enough uncertainty in the strong-interaction contribution to allow this to happen. (I am no expert here; in the corridors I found a rather broad spectrum of opinions on this from 
theorists more expert than me.) The obvious place to look is in the region of possible nonperturbative effects. The gluonic penguin contribution which topologically gives rise to these final states is shown in Fig. (1). The penguin is recumbent for a reason. The $c \bar{c}$ loop, which dominates the contribution ( $u \bar{u}$ is very small; $t \bar{t}$ simply provides the GIM cutoff at high mass scales), can have an absorptive part; $c \bar{c}$ occurs in real intermediate states, and the issue is how often it might annihilate into light quarks as shown. If it is going to do it in a big way, it has to be done more imaginatively than via single gluon annihilation.

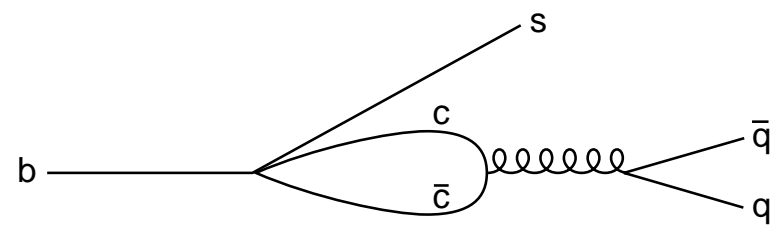

$\overline{\mathrm{q}}$

(a)

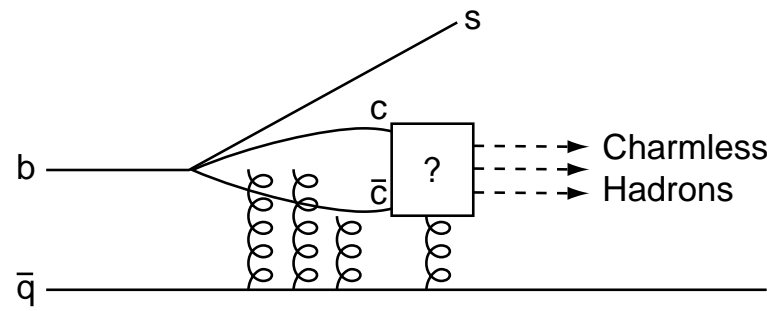

(b)

$5-97$
$8317 \mathrm{~A} 1$

Figure 1: Hadronic penguin contributions to $B$-decay: (a) short-distance, and (b) longdistance. Time runs from left to right.

If there is an enhancement of the annihilation, it is probable that it does occur for low $c \bar{c}$ virtual masses. The perturbative calculation should work at much higher masses, and common sense tells us that the nonperturbative effects should be largest when the charm system moves slowly. One nonper- 
turbative feature of this low mass system which is reasonably well-established is that its color quantum numbers fluctuate rapidly. This picture is the basis of the "color-evaporation" mechanism of onium production 38 , as well as a good candidate interpretation of rapidity-gap phenomenology in HERA electroproduction studies 39 . So although the $c \bar{c}$ may be initially color-octet, the probability at later times it is color singlet can be taken to be $1 / 9$. This mechanism probably is implemented via soft Coulomb-gluon exchanges with its local color environment (i.e. the spectator and $s$ quarks).

In the color-evaporation calculations 38 , when the $\bar{c} c$ has a perturbative mass less than $D \bar{D}$ threshold, $1 / 9$ of the events have the final state assigned to onium production. All the rest are assigned to open charm. This hypothesis agrees with hadroproduction, photoproduction, and $Z$-decay data on direct charmonium production very well. However, that agreement is insensitive to the question of how much of the subthreshold $c \bar{c}$ production emerges into open charm; perhaps some considerable fraction finds its way into charmless final states via annihilation. It is not so easy to come up with a mechanism for doing this, but we try by grasping at a straw, namely the large amounts of $\eta^{\prime}$ seen by CLEO 16, evocative of glueballs and/or instanton effects helping things along. If that were to be a clue, then lots of $\eta^{\prime}$ 's might be also seen in the final states of onium decays. With this stimulation (due to conversations with Steve Olsen), I checked out the PDG listings and found nothing in $\psi$ decays, but three remarkably large exclusive decay channels in $\eta_{c}$ decays: $\eta \pi \pi$, $\eta^{\prime} \pi \pi$, and $K \bar{K} \pi$. These three channels add up to about $15 \%$ of the total width. They all are obtainable from a six-fermion, instanton-induced effective vertex $E \cdot B(\bar{u} u \bar{d} d \bar{s} s)$, or even an 8 fermion vertex with $c \bar{c}$ appended. All of this seems evocative of communication of the pseudoscalar $\eta_{c}$ with $E \cdot B$ which communicates with the QCD axial anomaly, which communicates with instantons, all of which communicate with $\eta^{\prime}$ 's as well as pseudoscalar glueballs. I am told that something like this is advocated by Berkelman, who emphasizes $\eta_{c}-\eta^{\prime}$ mixing. In any case, I myself am interested in knowing more about those $\eta_{c}$ decay modes. Are they already studied and understood by someone?

So maybe the subthreshold, virtual $c \bar{c}$ systems also somehow communicate with these systems, leading with enhanced probability to charmless final states. As pointed out to me after my talk by a host of critics, this will have to be done in such a way as not to enhance the amount of $\eta_{c}$ in the $B$-decay final states. This, from experiment, is no larger than $1 \%$, and is said to be not out of line with conventional estimations.

Yes, all of this is pretty speculative, and maybe not warranted by the evidence that something is amiss. But it does lead to a few lines of study. One, independent of most details, is to check whether $\Lambda_{b}$ decays exhibit even 
more proclivity toward final states containing eta-primes than the $B$ mesons. If spectator effects matter in this business, the diquark spectator system for the $\Lambda_{b}$ may have a larger influence in driving a nonperturbative mechanism than the single quark spectator in the $B$. Anyway, perhaps a search for $\Lambda_{b} \rightarrow$ $\Lambda \eta^{\prime}$ is feasable. And, as stated earlier, the best way to normalize $b \rightarrow s+$ charmless is to measure the inclusive yield of $B \rightarrow K+$ anything when the $K$ momentum is large and when the $K$ does not come from a secondary-charm vertex. Also, for those who consider all of this as wallowing in minutiae, it should be remembered 40 that if there really is enhancement in such modes, which in $B_{s}$ decays are $s \bar{s}(c \bar{c})$ systems, then this can imply nonperturbative enhancement of $B_{s}$ mixing, in particular width-mixing. This in turn might open up new opportunities for CP-violation studies.

\section{Progress}

What follows will be personal reactions to what feels like recent progress. Since my perspective is based on a time scale of several years, it does not necessarily represent what has happened in the last year, half-year, month, or whatever the correct time quantum is that separates $B$-physics meetings like this one nowadays. The style will also be telegraphic; the reader is exhorted to go to the original sources for the real story:

1. Heavy-quarkeffective theory is working very well in the arena for which it was invented 11 , semileptonic $B$-decays, and the most important output is a precise determination of $V_{c b}$. There are many other reliable predictions of this method11, which, whatever its limits of applicability, provides a solid rock upon which to build a large body of theory.

2. CDF has made an impressive entry into the field 41,20, especially with respect to the high-quality determinations of decay modes containing a $\psi$, and the measurements of lifetimes. It bodes well for a very productive program in the next collider run at Fermilab.

3. Lifetime determinations of $B$-mesons and baryons are impressively accurate20, so much so that the subfield enters into the study of small lifetime differences.

4. The observation of the decay process $b \rightarrow s+\gamma$ is a remarkable experimental tour de force by itself 42 , and truly remarkable in attaining an accuracy that one can begin to look for new-physics effects at the $20 \%$ level. 
5. The experimental efforts on $b \rightarrow s+\gamma$ have been complemented-and complimented-by an extraordinary theoretical effort 43 , with two and three loop effects worked out. This is an arena where short-distance effects can with reasonable confidence be expected to prevail-although this is not fully guaranteed. But there now exists an impressive balance between the accuracy of the theoretical and experimental numbers, which are somewhere in between reasonable and marginal agreement. It may happen that, with a modest increase in accuracy on each side, the process may be elevated onto the trouble list. If so it will clearly be of enormous importance and interest.

6. Measurement of the charmless semileptonic decays is another experimental tour de force 4 , yielding a rather decent number for $V_{u b}$. The experiments now are $1 \frac{1}{2}$ generation, with exclusive channels being resolved and the beginnings of form-factor behavior being probed. The accuracy of $V_{u b}$ seems to me reasonably adequate already for the immediate future; it is $V_{t d}$ which would be nice to pin down more accurately.

7. The theory of the charmless semileptonic decays is maddeningly difficult. But I was very much impressed with a tasteful and serious attack by Burdman 4 , using the old-fashioned but powerful and appropriate dispersion-relation methodology. While so far the conclusions are modest, I hold out hope that another round along these lines will yield further benefits.

8. The most direct way of getting a better $V_{t d}$ is in the measurement of

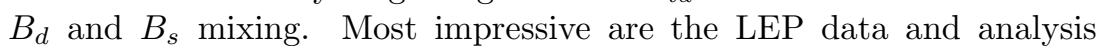
techniqu 46 , which are already at the edge of real observation of the mass difference at the value anticipated by theory, namely $x_{s}$ of order 10-40. I think that the level of accuracy attained exceeds what many anticipated in advance, thanks in part to the use of jet charge tagging and inclusive vertexing, as well as silicon detectors of improved accuracy. However, it appears that one will need to go beyond LEP to nail down the value of $x_{s}$.

9. There has been steady progress in the spectroscopy of the excited mesonic and baryonic $p$-wave states 25 - and perhaps higher. This is important to do for the charm states as well as bottom states; HQET is incisively tested by the comparisons, and the higher statistics for charm may allow finding the higher states more easily and determining the dynamics well enough that sharp predictions can be made for the $B$ and $\Lambda_{b}$ systems. I think it very important that the spectrum be fully filled in beyond the $p$-wave 
states and into the radial excitations and $d$-waves. Not only does this provide very important information for light-quark QCD spectroscopists, but it can also refine the single- $b$ flavor-tagging method $\left(B^{* *} \rightarrow B \pi^{ \pm}\right)$, so important for $\mathrm{CP}$ violation studies. In this regard it was especially interesting to see a candidate for the radial excitation of the $B$ reporte 25 .

10. The two-body rare decays of $B$ into $K \pi$ and $\pi \pi$, which seemed so hopelessly remote ten years ago, are now being seen 23 , with the two modes separated and with not uninteresting results, as already mentioned in the trouble list. There are many who eagerly await the not-yet-reported results on the ratio of $K^{+} \pi^{-}$to $K^{-} \pi^{+}$yields at CLEO.

11. The new results from CLEO on $B \rightarrow \eta^{\prime} K$ and $\eta^{\prime} K$ inclusive 6 are a fine experimental achievement, and also may represent an interesting issue for the theory: are these yields really "surprisingly large"?

12. The $D-\bar{D}$ mixing limits from Fermilab fixed-target charm experiment 4 are remarkably precise. While standard-model expectations are well below the data, these still probe the new-physics frontier in interesting ways 4849.

13. The $B_{c}$ candidate from ALEPH arrives ahead of schedule 5 , but is welcome nevertheless. Now and then such things do arrive ahead of schedule; in any case this event is a harbinger of things to come. I also look forward to the first observation of doubly charmed baryons.

14. As already mentioned, the unitarity triangle exhibited by Forty 6 was most impressive in its purported accuracy and internal consistency. If only the theoretical uncertainties were less!!

15. Reports from the lattice world showed steady improvement in the accuracy of the old measurements and the scope of lattice measurements attempted. However, the quenched approximation, together with the difficulties in implementing the approximate chiral symmetry in QCD, remains a serious obstacle, and any and all attempts to overcome it are extremely welcome.

16. Progress reports from the new generation of kaon $\mathrm{CP}$ experiment 51.52 .53 bode well for a decisive conclusion from that long and extremely demanding program. Perhaps the first solid evidence for $\mathrm{CP}$ violation originating from the CKM mixing will emerge from this source. 
In addition to these results, there has been much progress in experimental technique. I rank the high-rate charm experiments E791/E687 from Fermilab as very important, especially since they pave the way for similar experiments at the hadron colliders. And in the realm of analysis technique, there were impressive new methods exhibited. The use at CLEO of neural-net methods 42 may not be the very first application, but it seems to me the first time a claim of a major experimental result is largely based upon that technique. Also, the use of Fisher discriminants and multidimensional likelihood analyses, etc., again at Cornell 1623, in the determination of the $B$ branching ratios into $K \pi$ and $\pi \pi$ appears to me to be another escalation in statistical sophistication.

Another landmark analysis has come from ALEPH, in the intraduction of the "amplitude method" for the interpretation of $B_{s}$ mixing data 4 . This technique is already becoming a standard for the field. I am not sure that the quality of the present limits on $B_{s}$ mixing derives directly from this analysis method, but if nothing else it creates clarity in assessing the experimental situation.

On the theoretical side, the compendium of suggestions for CP-violation observations continues to grow. A list culled from the talks, no doubt incomplete, includes the following:

1. Rate asymmetries in the $B$ decays to $K+$ charmless-nonleptonic final states, either in exclusive channels or even inclusively 54.13 .

2. CP violation in $B \rightarrow \phi K_{s}$, a special case of the above 55 .

3. Rate asymmetries 48 in $D \rightarrow \rho \pi$.

4. Rate asymmetries 56 in $B \rightarrow D^{0} K^{-}$, with the $D^{0} \rightarrow K \pi$ of either strangeness.

5. $\mathrm{CP}$ violation studies which exploit the difference in lifetimes of the two $B_{s}$ mass eigenstates 40 .

Again, I exhort, I hope unnecessarily, experimentalists to check everywhere for $\mathrm{CP}$ violating effects. I can imagine a situation where the first observation occurs in a subregion of a Dalitz plot for a messy final state (of large branching ratio), where strong final-state phase shifts conspire to create a maximal effect, much to the surprise of conservative theorists. Lucky maximal effects might after all provide quite good measurements of CKM CP-violating parameters via the setting of lower bounds. Theory might end up being data-driven, rather than the data being theory-driven. 


\section{Prospects}

The present progress derives from technological advances. And the technological revolution will continue, with no saturation in sight. The new electronpositron machines are promised to operate at luminosities of $10^{33}-10^{34}$, values that seemed ten years ago most unreasonable. This will not be easy, and new buzzwords like PEI (photoelectric instability) and FII (fast ion instability) appear in the talks 57 as sobering reminders of the difficulties. On the hadron-hadron front, at the LHC 55 (and perhaps with a new opportunity at $\mathrm{C} 0$ at Fermilab 9 ) there should be for the first time at least one dedicated $B$-physics detector existing in hadron-collider mode. The enormous yield of $b$ 's and c's produced at hadron colliders will create opportunities which are off-scale relative to what exists in present data.

In order to exploit the high rates and luminosities, there should - and almost certainly will be - major advances in detection capability. The emergence of micropixel detectors of silicon or diamond 60 will, in my opinion, be a technological advance comparable to that created by the silicon microstrips. The high rates and bandwidth attained in the Fermilab charm experiments will be augmented even more in the next-generation detectors. I found it amusing to hear Brad Cox describe the LHC-B plan to run at luminosities of $2 \times 10^{32}$ as "conservative"; $\mathrm{CDF}$ and D0 are near meltdown as these rates are approached, and such multi-megahertz rates even in fixed-target mode are not at all commonplace. Nevertheless it is an accurate expression of conventional wisdom.

The trigger algorithms of the future are again of unprecedented sophistication. The "track triggers" of CDF, already being prepared for the next run 61, are designed to partially reconstruct at Level II $B \rightarrow h^{+} h^{-}$. This was not in the game plan ten years ago at all. And the successes of ring-imaging Cerenkov counters make their introduction into hadron collider detectors most welcome: Cerenkov identification has been completely absent from all such detectors since the ISR days, and it is time to bring it back.

There is also an impressive array of new detectors. For the electronpositron machines there is the BBC trio 62: BABAR, BELLE, and CLEO. CDF and D0 will have powerful upgrades for their next run. And, there will be novel devices such as HERA-B 3 . Ten years agot I looked toward a sophisticated high-rate fixed target experiment as the future of the field. While it had the disadvantage of low signal/background, it did not suffer the colliderexperiment disadvantage of having a circulating beam and beampipe in the middle of the detector. HERA-B is the closest thing being built to what I had in mind, although it manages to synthesize the disadvantages of both approaches. But even so, I am an enthusiast: it is an adventurous, powerful 
approach, and one which will face directly the problems to be faced later at the LHC with LHC-B, or at the TeVatron with BTeV.

In addition to these collider initiatives, there is the innovative initiative E871 at Fermilab 53 to look for $\mathrm{CP}$ violation in hyperon decays. It again pushes the high-rate frontier in interesting ways. And finally there are the experiments underway to measure $\epsilon^{\prime} / \epsilon$, sure to take their place in the history of the field as classics of experimental technique 51,53.

So with this array of opportunities, it seems clear that if the standardmodel CKM scenario for CP violation is correct, the present program should provide a high-precision, quite complete description. On the other hand, the next 20 years may be less programmatic than the last 20. And if the picture is not correct, then the new facilities should have enough flexibility to respond to an unpredictable change in emphasis.

\section{Conclusion}

The progress in this field has been extraordinary, and the prospects are extremely bright. It is due to the efforts of everyone in the CP-business that this has come about. I offer my most warm and sincere congratulations.

\section{Acknowledgments}

I thank the many participants who have helped to minimize the inaccuracies in this summary. Also on behalf of all participants I thank Tom Browder, Sandip Pakvasa, and their excellent staff for making this meeting so pleasant and successful. In particular, the organization of the meeting by subject-matter is not an easy task, requiring a delicate touch, and this has been accomplished in a splendid manner.

\section{References}

1. J. Bjorken, Proceedings of the 5th Moriond Workshop, "Flavor Mixing and CP Violation," La Plagne, France, January 1985, ed. J. Tran Thanh Van (Editions Frontieres, Singapore, 1985), p. 455.

2. N. Isgur and M. Wise, Phys. Lett. B232, 113 (1989); B237, 527 (1990).

3. In my talk at Moriond (Ref. 1), some time was devoted to exhibiting the rough specifications of what was required.

4. See, for example, the report of the Fermilab Physics Advisory Committee on its specifications for the future use of the $C \varnothing$ collision region in the 
Fermilab TeVatron: http://www.fnal.gov/directorate/

program_planning/phys_adv_com/Aspen96Web.html

5. P. Frampton, these proceedings.

6. R. Forty, these proceedings.

7. L. Wolfenstein, these proceedings.

8. H. Fritzsch, hep-ph/9605388.

9. B. Stech, Phys. Lett. 130B, 189 (1983).

10. This in particular implies, contrary to the hypothesis of "Fritzsch texture," that there is not much renormalization of the strange-quark mass. I have been involved in Stech's hypothesis for quite some time (J. Bjorken, Proceedings of the Eighteenth SLAC Summer Institute on Particle Physics, July, 1990, SLAC-REPORT-378, ed. J. Hawthorne (SLAC), p. 167, and occasional seminars), and I still work on it.

11. M. Neubert, these proceedings. See also M. Neubert, Phys. Rev. 245, 259 (1994).

12. I. Dunietz, these proceedings. See also I. Dunietz et al., hep-ph/9612421.

13. A. Kagan, these proceedings.

14. H. Yamamoto, these proceedings.

15. See, for example, M. Ciuchini et al., Phys. Lett. B334, 137 (1994), W. F. Palmer and B. Stech, Phys. Rev. D48, 4174 (1993).

16. B. Behrens, these proceedings.

17. M. Bauer, B. Stech, and M. Wirbel, Z. Phys. C34, 103 (1987).

18. Since this meeting, responses from theorists have already appeared: Dr. Atwood and A. Soni, hep-ph/9704357; I. Halperin and A. Zhitnitsky, hep-ph/9704412; Wei-Shu Hou and B. Tseng, hep-ph/9705304.

19. J. Richman in Proceedings of ICHEP96, Warsaw, Poland, July 1996.

20. T. Junk, these proceedings; see also Ref. 22.

21. J. Rodriguez, these proceedings.

22. B. Stech, these proceedings.

23. J. Alexander, these proceedings.

24. A. Falk, these proceeedings.

25. M. Feindt, these proceeedings.

26. M. Olsson, these proceedings.

27. M. Luke, these proceedings.

28. K. Kreuter, these proceedings.

29. A. Ryd, these proceedings.

30. N. G. Deshpande, these proceedings. See also N. G. Deshpande and X.G. He, Phys. Rev. Lett. 74, 26 (1995); M. Gronau et al., Phys. Rev. D52, 6374 (1995); M. Gronau and D. London, Phys. Rev. Lett. 65, 3381 (1990). 
31. G. Hanson et al., Phys. Rev. Lett. 35, 1609 (1975).

32. E. Poggio, H. Quinn, and S. Weinberg, Phys. Rev. D13, 1958 (1976).

33. J. Bjorken, Nucl. Phys. B11 (Proc. Suppl), 325 (1989).

34. See for example A. Manohar, Proceedings of the Tenth Lake Louise Winter Institute, "Quarks and Colliders," February, 1995, ed. A. Ashbury et al. (World Scientific, 1995), p. 274.

35. J. Gasser and H. Leutwyler, Nucl. Phys. B250, 465 (1985).

36. T. Schafer and E. Shuryak, hep-ph/9610451 and references therein.

37. D. Diakonov, nucl-th/9603023 and references therein.

38. O. Eboli, E. Gregores, and F. Halzen, hep-ph/9611258 and references therein.

39. W. Buchmuller and A. Hebecker, Phys. Letts. B355, 573 (1995).

40. R. Fleischer, these proceedings.

41. J. Lewis, these proceedings.

42. J. Ernst, these proceedings. See also M. S. Alam et al. (CLEO Collaboration), Phys. Rev. Lett. 74, 2885 (1995).

43. C. Greub, these proceedings, and C. Greub, T. Hurth and D. Wyler, Phys. Lett. B380, 385 (1996); Phys. Rev. D54, 3350 (1996); K. G. Chetyrkin, M. Misiak and M. Münz, hep-ph/9612313; K. Adel and Y.-P. Yao, Phys. Rev. D49, 4945 (1994).

44. R. Poling, these proceedings.

45. G. Burdman, these proceedings; also G. Burdman and J. Kambor, Phys. Rev. D55, 2817 (1997).

46. A. Ouraou, these proceedings.

47. M. Purohit, these proceedings. See also E. M. Aitala et al., (FNAL E791), Phys. Rev. Lett. 77, 2384 (1996).

48. E. Golowich, these proceedings.

49. Z.-Z. Xing, these proceedings.

50. S. Hashimoto, these proceedings.

51. T. Yamanaka, these proceedings.

52. A. Schopper, these proceedings.

53. M. Calvetti, these proceedings.

54. X.-G. He, these proceedings; T. Browder et al., hep-ph/9705320.

55. M. Worah, these proceedings.

56. D. Atwood, these proceedings; see also I. Dunietz, "B Decays" (ed. S. Stone), World Scientific (1994), p. 550.

57. S. Kurokawa, these proceedings.

58. B. Cox, these proceedings.

59. S. Stone, these proceedings.

60. H. Kagan, these proceedings. 
61. A. Maciel, these proceedings.

62. P. Harrison, these proceedings.

63. K. Ehret, these proceedings. 